\title{
Recent developments in tropical cyclone analysis using observations and high resolution models
}

\author{
U. C. Mohanty $\cdot$ Dev Niyogi $\cdot$ K. V. J. Potty
}

Received: 10 January 2012/Accepted: 10 January 2012/Published online: 25 January 2012

(C) Springer Science+Business Media B.V. 2012

Affecting scores of people with seemingly insurmountable and unmanageable costs, tropical cyclones (TCs) are one of the most devastating extreme weather events. In this special issue, we highlight and summarize some of the recent studies that make use of detailed observations and high-resolution numerical models to analyze tropical cyclones from their genesis to post-landfall impacts. Focused around TC activity in the Atlantic, North Indian, and Western Pacific basins, the different papers in this issue contribute to an increased understanding of TCs and to our scientific and operational ability to predict their nature and characteristics.

Opening the issue is a comprehensive review from the India Meteorological Department operational forecasters Mohapatra, Bandyopadhyay, and Tyagi on TC climatology over the North Indian Ocean (NIO). The best track parameters related to the position and intensity of a TC were analyzed from the pre-1877 era to 2010. This paper provides an authoritative summary on the errors and uncertainty associated with TC tracks and intensity and offers insight into the methodology, trends, and future consideration for improved TC analysis over the NIO. Mohanty et al. extend this perspective and review storm characteristics over the Bay of Bengal and the Arabian Sea under two epochs-one until 1950 and another from 1950 onward. Their analysis of the two epochs, corresponding to a so-called past cooling period and current warming period, showed that in recent decades, there is a statistically significant increase in the number of severe cyclones, particularly over the Bay of Bengal in the post-monsoon season. The Mohanty et al. study also finds an overall increase in severe cyclones in the western Arabian Sea and southern Bay of Bengal.

From the modeling perspective, Osuri et al. reviewed the performance of the Advanced Research Weather Research and Forecasting (WRF-ARW) model over NIO. They

U. C. Mohanty $(\bowtie)$

Indian Institute of Technology Delhi, New Delhi, India

e-mail: ucmohanty@gmail.com

D. Niyogi

Purdue University, West Lafayette, IN, USA

K. V. J. Potty

Regional Integrated Multi-Hazard Early Warning System, Pathumthani, Thailand 
simulated five recent TCs with a combination of different parameterization schemes to compare the forecast with those from the different operational centers for the 2008 hurricane season. Their results indicate that the YSU boundary layer scheme with the KainFritsch convection parameterization can be a preferred configuration when simulating TCs over NIO with the ARW modeling system. Using a similar model configuration as recommended in Osuri et al., an extended analysis for the 2007-2010 severe tropical cyclones over the Bay of Bengal was conducted by Raju et al. Their study also compared and contrasted the model results with different observations, analysis, and satellite estimates available over the Bay of Bengal region. A mean track error at landfall of approximately $100 \mathrm{~km}$ seems to be prevalent in most of the model experiments for the Bay of Bengal region. Potty et al. extend the analysis using the same WRF-ARW model configuration with one-way nesting for simulating typhoons over the Western Pacific Ocean and South China Sea. Their study concludes that the resolution plays a relatively small role in improving the track forecasts, and an error between 100 and $200 \mathrm{~km}$ for 24 and $48 \mathrm{~h}$ of model simulations remained.

Liou and Sashegyi discuss the development and application of a TC initialization method using the US Naval Research Laboratory (NRL) variational data assimilation (VAR) system. They investigated the impact of using synthetic observations, vortex relocation, and 3DVAR within a hurricane model. They found that each of these assimilation approaches has a positive impact on model performance related to track forecast and intensity, with the vortex relocation producing the largest improvement. Bhaskar Rao and Tallapragada used three models - the Hurricane WRF (HWRF), WRF-ARW, and the Fifth generation Mesoscale Model (MM5) - to simulate two Bay of Bengal TCs-Sidr (2007) and Nargis (2008). They found HWRF with the vortex initialization procedure produces a superior forecast for the region. Similarly, Pattanayak et al. analyzed TC Mala (2006) that struck the Bay of Bengal as a case study and conducted simulation experiments with HWRF. Their results showed that HWRF is capable of reproducing the TC features with reasonable accuracy. Yeh et al. continue this evaluation of the HWRF model but for the 2008 hurricane season in the Atlantic Basin. They compared the experimental HWRF performance with that of the NOAA operational models. This study showed that the HWRF performed comparably to the operational NOAA Geophysical Fluid Dynamics Laboratory (GFDL) model with only limited improvement in the track forecast over the Global Forecast System (GFS) despite enhanced resolution. The study also highlights the need for better metrics in hurricane model evaluations.

A number of papers also sought to understand the role of planetary boundary layer (PBL) and surface processes on storm characteristics. Ramakrishna et al. studied the role of the planetary boundary layer for the simulation of tropical cyclone Nargis (2008). Their study indicates that the KF2 for cumulus convection and Eta MY PBL processes produces the most intensive cyclone both in 90 and $30 \mathrm{~km}$ resolution. However, the Blackadar PBL scheme also provides similar results in $30 \mathrm{~km}$ resolution. Yeh et al. studied Typhoon Haitang (2005) that made landfall over the Central Mountain Range of Taiwan. Their analysis shows that terrain interaction induces a cyclonic vortex that can interact with the typhoon, causing a looping track prior to landfall over Taiwan. Sadhuram et al. studied the role of offshore eddies in the intensification of TC Aila (2009) in the Bay of Bengal. Their analysis identifies the role of surface heat flux induced by the oceanic eddy in modifying storm intensity before landfall.

Studies by Kishtawal et al., Kellner et al., and Bozeman et al. investigated the issue of post-landfall TC characteristics. Kishtawal et al. studied TC landfall over the Atlantic Basin for the post-satellite era to understand the role of land surface/soil characteristics on 
cyclone decay. Using a data adaptive genetic algorithm approach, they found that soil bulk density (heat capacity) has a dominant impact on inland cyclone decay. They tested this relation with recent landfalling hurricanes and show good predictive skill for the statistical model while also highlighting the inability of WRF-ARW to correctly capture these inland processes. Bozeman et al. studied Tropical Storm Fay (2008) that developed a storm central eye after landfall over south Florida. Using the HWRF model to diagnose this anomalous intensification, Bozeman et al. concluded that Florida's Lake Okeechobee and the Everglades may have provided surface feedback as the storm approached these land surface features to intensify and form a storm eye over land rather than decaying over land. In a related study, Tropical Storm Erin (2007) intensified and showed hurricane-like characteristics hundreds of $\mathrm{km}$ after landfall. Kellner et al. reviewed observations, operational analysis, and conducted modeling studies to investigate the hypothesis pertaining to the role of anomalously wet antecedent soil conditions leading to the re-intensification of Tropical Storm Erin (2007). These studies call for reassessing the representation of land surface processes within hurricane models.

Concluding the issue is a paper by Mohapatra et al. that offers a classification scheme for the purpose of cyclone disaster management in the Bay of Bengal. Aimed at identifying cyclone-prone districts, the researchers based their classes on the degree of hazard and labeled the various coastal districts of India adjoining the Bay of Bengal from very high to low cyclone proneness.

The sixteen papers in this special issue thus provide evidence that the assimilation of observational datasets from satellite, radar, and aircraft reconciliation plays an important role in improving cyclone simulations particularly as the system approaches landfall. Reducing the persistent uncertainties in current models for simulating track and intensity despite finer resolution (grid spacing) remains a challenge. The different studies also highlight the need for improved metrics for use in evaluating different models and the potential for improvements when introducing newer data or model enhancements. The role of land surface characteristics and their influence on the intensity and structure of landfalling tropical cyclones also remain to be further investigated along with the impact of land surface data assimilations for improving their prediction. Finally, the vulnerability of regional landscapes to TCs and the resilience or the infrastructural preparedness need to be studied as an end-to-end approach from a forecasting to societal response perspective. 